\title{
Discretization of Toroidal Soliton Equations
}

\author{
Yasuhiro OHTA
}

Information Engineering, Graduate School of Engineering, Hiroshima University, 1-4-1

Kagamiyama, Higashi-Hiroshima 739-8527, Japan

E-mail: ohta@kurims.kyoto-u.ac.jp

This paper is part of the Proceedings of SIDE V;

Giens, June 21-26, 2002

\begin{abstract}
We propose a way of discretization for the soliton equations associated with the toroidal Lie algebra based on the direct method. By the discretization, the symmetry of the system is modified so that the discrete time evolutions are no longer compatible with the original continuous ones. The solutions of the discrete soliton equations are explicitly given in terms of the Casorati determinants.
\end{abstract}

\section{Introduction}

As is well known, the hierarchies of soliton equations have the symmetries described by the infinite dimensional Lie algebras, affine or toroidal. All of the KP hierarchies are derived from the symmetries of affine Lie algebras[6], while the toroidal Lie algebras are associated with the $2+1$ dimensional generalization of the $1+1$ dimensional reduced $\mathrm{KP}$ hierarchies[1, 5, 4, 7]. For example, the $2+1$ dimensional KdV equation[3]

$$
u_{t}=u_{x x y}+2 u u_{y}+u_{x} \int^{x} u_{y} d x
$$

and the $2+1$ dimensional nonlinear Schrödinger equation[12]

$$
i u_{t}=u_{x y}+u \int^{x}\left(|u|^{2}\right)_{y} d x
$$

belong to the 2-toroidal hierarchy. In the above equations, the variable $x$ is derived from the action of the usual affine part of the toroidal Lie algebra, while the evolutions in $y$ and $t$ are induced by the action of the genuine toroidal part. The weight of $u$ and the relative weight of $y$ and $t$ are balanced with that of $x$, so we have two freedoms to determine the weights for all of the variables, the weight of $x$ itself and the absolute value of weight of $y$ (or $t$ ), which is one of the features of the 2-toroidal hierarchy. Another famous example is the self-dual Yang-Mills equation[11]

$$
\left(J_{y} J^{-1}\right)_{\bar{y}}+\left(J_{z} J^{-1}\right)_{\bar{z}}=0
$$


which has the 3-toroidal Lie algebra symmetry, where we have three freedoms to balance all of the weights.

The integrable discretizations for the equations in KP hierarchies, i.e. affine case, have been investigated widely and deeply for many years from various standing points of view. For example, the Miwa transformation[9]

$$
\begin{aligned}
& \partial_{k}=a \partial_{x_{1}}+\frac{a^{2}}{2} \partial_{x_{2}}+\frac{a^{3}}{3} \partial_{x_{3}}+\cdots \\
& \partial_{l}=b \partial_{x_{1}}+\frac{b^{2}}{2} \partial_{x_{2}}+\frac{b^{3}}{3} \partial_{x_{3}}+\cdots \\
& \partial_{m}=c \partial_{x_{1}}+\frac{c^{2}}{2} \partial_{x_{2}}+\frac{c^{3}}{3} \partial_{x_{3}}+\cdots
\end{aligned}
$$

can always produce integrable discrete analogues for a given continuous soliton equation in KP hierarchies, because the discrete evolutions in $k, l, m, \cdots$ are nothing but the chains of Bäcklund transformations.

On the other hand, nothing has been studied for the integrable discretization in the case of toroidal Lie algebra. In this paper, we investigate how to discretize the soliton equations associated with the toroidal Lie algebra keeping integrability and propose a way of discretization by using the Hirota's direct method of soliton theory. The discrete equations are bilinearized by introducing the auxiliary independent variables which come from affine symmetry and the solutions are directly constructed in the form of Casorati determinants.

\section{Discretization for toroidal case}

In the KP case, i.e. affine case, the solution depends on all of the continuous independent variables $x_{1}, x_{2}, x_{3}, \cdots$ and the discrete ones $k, l, m, \cdots$ simultaneously, and all of the flows induced by these continuous and discrete variables are compatible on the space of solution. Both the continuous and the discrete flows are described by the action of the same affine Lie algebra. Thus in which form we write down the equation is just a matter of choice of the variables. If we choose the continuous independent variables, we get the (hierarchy of) continuous soliton equations, and if we choose the discrete variables, we get the discrete analogues of soliton equations, and of course by choosing both continuous and discrete variables, we get the partial differential difference equations. Therefore in the KP case, we can easily derive integrable discrete analogues for a given continuous equation, because it is sufficient to change the choice of independent variables without changing the structure of the space of solution.

On the other hand in the case of toroidal symmetry, the solution depends on the continuous and discrete variables, $x_{1}, x_{2}, x_{3}, \cdots$ and $k, l, m, \cdots$ which come from the affine part and the continuous variables only, $y_{1}, y_{2}, y_{3}, \cdots$ which come from the genuine toroidal part. The point is that there is no discrete compatible flow associated with the genuine toroidal part because all of the Bäcklund transformations can be described by the affine part only and no discrete variable associated with $y_{1}, y_{2}, y_{3}, \cdots$ can be introduced. For example, there is no Miwa transformation for $y_{1}, y_{2}, y_{3}, \cdots$. The essential difficulty 
to construct discrete anologues for the case of toroidal Lie algebra comes from the above fact.

In order to discretize $y_{1}, y_{2}, y_{3}, \cdots$, we give up keeping compatibility of all of the flows and break the affine symmetry. In other words, in order to introduce the discrete independent variables $K, L, M, \cdots$ associated with the toroidal part, with keeping the continuous toroidal variables $y_{1}, y_{2}, y_{3}, \cdots$, we modify the structure of the space of solution so that the original affine symmetry is killed and only $A_{\infty}^{(1)}$ symmetry survives. In the following sections, we are going to explain concretely how to modify by taking a typical example of the soliton equations with toroidal symmetry.

\section{Modified Bogoyavlenskii-Schiff equation}

The modified Bogoyavlenskii-Schiff equation[2, 10]

$$
4 u_{y_{3}}=u_{x_{1} x_{1} y_{1}}-4 u^{2} u_{y_{1}}-2 u_{x_{1}} \int^{x_{1}}\left(u^{2}\right)_{y_{1}} d x
$$

which has $s l_{2}^{\text {tor }}$ symmetry, is bilinearized into the bilinear form

$$
\begin{aligned}
& D_{x_{1}}^{2} g \cdot f=0 \\
& \left(D_{y_{1}} D_{x_{1}}^{2}-4 D_{y_{3}}\right) g \cdot f=0 \\
& \left(D_{x_{1}}^{3}-4 D_{x_{3}}\right) g \cdot f=0
\end{aligned}
$$

through the dependent variable transformation

$$
u=\left(\log \frac{g}{f}\right)_{x_{1}}
$$

where $x_{3}$ is the auxiliary independent variable. The evolutions along the variables $x_{k}$ are described by the affine symmetry $A_{1}^{(1)}$ and those for $y_{k}$ are induced by the genuine toroidal part.

The solution of the bilinear equations is given in the form of the Wronski determinant

$$
f=\operatorname{det}\left(\varphi_{i}^{(j-1)}\right) \quad g=\operatorname{det}\left(\varphi_{i}^{(j)}\right)
$$

where $\varphi_{i}^{(j)}$ are arbitrary functions satisfying the following dispersion relations associated with the affine part and genuine toroidal part respectively

$$
\begin{aligned}
& \partial_{x_{k}} \varphi_{i}^{(j)}=\varphi_{i}^{(j+k)} \\
& \partial_{y_{3}} \varphi_{i}^{(j)}=\partial_{y_{1}} \varphi_{i}^{(j+2)}
\end{aligned}
$$

and also satisfying the condition of 2-reduction

$$
\varphi_{i}^{(j)} \approx \varphi_{i}^{(j+2)}
$$

where $\approx$ means 'equal up to gauge', i.e., equal up to multiplication of exponential function of linear combination of the independent variables. 


\section{Discrete modified Bogoyavlenskii-Schiff equation}

In the above modified Bogoyavlenskii-Schiff equation, discretizing the affine variables $x_{k}$ is quite simple and straightforward[8], because we can just apply the Miwa transformation on $x_{k}$ and replace the continuous affine variables by the compatible discrete ones. Our purpose here is to discretize the genuine toroidal variables $y_{k}$.

In order to discretize $y_{k}$, the affine symmetry $A_{1}^{(1)}$ is modified to $A_{\infty}^{(1)}$. Under this modification, we will have all of the even and odd affine variables $X_{1}, X_{2}, X_{3}, \cdots$, instead of just odd ones $x_{1}, x_{3}, x_{5}, \cdots$ for $A_{1}^{(1)}$. Let us denote the discrete independent variables corresponding to $y_{k}$ as $n_{k}$, then this modification is simply given by introducing some shifts into the components of determinant in the following manner.

$$
f_{n_{1} n_{3}}=\operatorname{det}\left(\varphi_{i}^{(j-1)}\left(n_{1}-j, n_{3}+j\right)\right) \quad g_{n_{1} n_{3}}=\operatorname{det}\left(\varphi_{i}^{(j)}\left(n_{1}-j, n_{3}+j\right)\right)
$$

where $\varphi_{i}^{(j)}$ are arbitrary functions satisfying the dispersion relations of continuous affine and discrete toroidal types

$$
\begin{aligned}
& \partial_{X_{k}} \varphi_{i}^{(j)}\left(n_{1}, n_{3}\right)=\varphi_{i}^{(j+k)}\left(n_{1}-k, n_{3}+k\right) \\
& \Delta_{3} \varphi_{i}^{(j)}\left(n_{1}, n_{3}\right)=\Delta_{1} \varphi_{i}^{(j+2)}\left(n_{1}, n_{3}\right)
\end{aligned}
$$

where $\Delta_{k}$ is the backward difference operator with respect to $n_{k}$ defined as $\Delta_{k} F\left(n_{k}\right)=$ $F\left(n_{k}\right)-F\left(n_{k}-2\right)$. And $\varphi_{i}^{(j)}$ should also satisfy

$$
\varphi_{i}^{(j)}\left(n_{1}, n_{3}\right) \approx \varphi_{i}^{(j+2)}\left(n_{1}, n_{3}\right)
$$

We should note that this is not the condition of 2-reduction, because the components of determinant have shifted indices. Therefore the original $A_{1}^{(1)}$ flows are no longer compatible and the solution has only $A_{\infty}^{(1)}$ symmetry as the affine part.

The bilinear equations satisfied by the above Casorati determinants with the shifted indices of components are

$$
\begin{aligned}
& \left(D_{X_{1}}^{2}-D_{X_{2}}\right) g_{n_{1}-1, n_{3}+1} \cdot f_{n_{1} n_{3}}=0 \\
& \left(D_{X_{1}}^{2}+D_{X_{2}}\right) g_{n_{1}+1, n_{3}-1} \cdot f_{n_{1} n_{3}}=0 \\
& g_{n_{1}+1, n_{3}+1} f_{n_{1} n_{3}}-g_{n_{1}+1, n_{3}-1} f_{n_{1}, n_{3}+2}=\frac{1}{2}\left(D_{X_{1}}^{2}-D_{X_{2}}\right) g_{n_{1}+1, n_{3}+1} \cdot f_{n_{1} n_{3}} \\
& g_{n_{1} n_{3}} f_{n_{1}+1, n_{3}+1}-g_{n_{1}, n_{3}+2} f_{n_{1}+1, n_{3}-1}=\frac{1}{2}\left(D_{X_{1}}^{2}+D_{X_{2}}\right) g_{n_{1} n_{3}} \cdot f_{n_{1}+1, n_{3}+1}
\end{aligned}
$$

Here the later two bilinear equations are new. Finally by introducing the dependent variable transformation

$$
\begin{gathered}
u_{n_{1} n_{3}}= \begin{cases}\left(\log \frac{g_{n_{1}-1, n_{3}}+1}{f_{n_{1} n_{3}}}\right)_{X_{1}} & n_{1}, n_{3}: \text { even } \\
\left(\log \frac{f_{n_{1}-1, n_{3}+1}}{g_{n_{1} n_{3}}}\right)_{X_{1}} & n_{1}, n_{3}: \text { odd }\end{cases} \\
Q_{n_{1} n_{3}}= \begin{cases}\left(\log \frac{g_{n_{1}+1, n_{3}}}{g_{n_{1}-1, n_{3}}}\right)_{X_{1}} & n_{1}: \text { even, } n_{3}: \text { odd } \\
\left(\log \frac{f_{n_{1}+1, n_{3}}}{f_{n_{1}-1, n_{3}}}\right)_{X_{1}} & n_{1} \text { odd }, n_{3}: \text { even }\end{cases}
\end{gathered}
$$


we obtain the discrete modified Bogoyavlenskii-Schiff equation

$$
\begin{aligned}
& \Delta e^{\int^{X_{1}} \Delta_{3} u_{n_{1} n_{3}} d X_{1}}=\left(Q_{n_{1} n_{3}}\right)_{X_{1}}-Q_{n_{1} n_{3}}\left(u_{n_{1}+1, n_{3}}-u_{n_{1}, n_{3}-1}\right) \\
& \Delta Q_{n_{1} n_{3}}=\Delta_{1} u_{n_{1}+2, n_{3}}
\end{aligned}
$$

where $\Delta$ is the difference operator defined as $\Delta F\left(n_{1}, n_{3}\right)=F\left(n_{1}, n_{3}+1\right)-F\left(n_{1}+1, n_{3}\right)$.

\section{Concluding remarks}

The way to discretize the genuine toroidal independent variables of the soliton equations associated with the toroidal Lie algebra symmetry was given by modifying the structure of the space of solution, i.e., by introducing the shifts of discrete variables into the components of the Casorati determinant solutions. Since the structure is changed, the symmetry of the system is not preserved, which is quite different from the case of usual discretization of affine flows. This method of discretization is applicable to any 2-toroidal Lie algebras but not to the 3-toroidal case, thus the discretization of the self-dual Yang-Mills equation would be a future work.

The author thanks to Dr. Kakei for valuable discussions.

\section{References}

[1] Billig Y, An Extension of the Korteweg-de Vries Hierarchy Arising from a Representation of a Toroidal Lie Algebra, J. Algebra 217 (1999), 40-64.

[2] Bogoyavlenskii O I, Overturning Solitons in New Two-Dimensional Integrable Equations, Izv. Akad. Nauk SSSR Ser. Mat. 53 (1989), 243-257.

[3] Calogero F, A Method to Generate Solvable Nonlinear Evolution Equations, Lett. Nouvo Cimento 14 (1975), 443-447.

[4] Ikeda T and Takasaki K, Toroidal Lie Algebras and Bogoyavlensky's (2+1)-Dimensional Equation, Internat. Math. Res. Notices No. 7 (2001), 329-369.

[5] Iohara K, Saito Y and Wakimoto M, Hirota Bilinear Forms with 2-Toroidal Symmetry, Phys. Lett. A 254 (1999), 37-46.

[6] Jimbo M and Miwa T, Solitons and Infinite-Dimensional Lie Algebras, Publ. RIMS, Kyoto Univ. 19 (1983), 943-1001.

[7] Kakei S, Ikeda T and Takasaki K, Hierarchy of (2+1)-Dimensional Nonlinear Schrödinger Equation, Self-Dual Yang-Mills Equation, and Toroidal Lie Algebras, nlin.SI/0107065.

[8] Kakei S and Ohta Y, A Differential-Difference System Related to Toroidal Lie Algebra, J. Phys. A 34 (2001), 10585-10592.

[9] Miwa T, On Hirota's Difference Equations, Proc. Jpn. Acad. 58A (1982) 9-12.

[10] Schiff J, Integrability of Chern-Simons-Higgs Vortex Equations and a Reduction of the SelfDual Yang-Mills Equations to Three Dimensions, in Painlevé Transcendents, Their Asymptotics and Physical Applications, Editors: Levi D and Winternitz P, Plenum Press, New York, 1992, 393-405. 
[11] Yang C N, Condition of Self-Duality for SU(2) Gauge Fields on Eudulidean Four-Dimensional Space, Phys. Rev. Lett. 38 (1977), 1377-1379.

[12] Zakharov V E, The Inverse Scattering Method, in Solitons, Editors: Bullough R K and Caudrey P J, Springer-Verlag, Berlin, Heidelberg, 1980, 243-285. 